\title{
Manufacturing and Integration Status of the JWST OSIM Optical Simulator
}

Joe Sullivan ${ }^{\mathrm{a}}$, Bill Eichhorn ${ }^{\mathrm{b}}$, Rob von Handorf $\mathrm{f}^{\mathrm{a}}$, Derek Sabatke ${ }^{\mathrm{a}}$, Nick Barr ${ }^{\mathrm{a}}$, Rich Nyquist ${ }^{\mathrm{a}}$, Bob Pederson ${ }^{\mathrm{a}}$, Rick Bennett ${ }^{\mathrm{a}}$, Paul Volmer ${ }^{\mathrm{a}}$, Dave Happs ${ }^{\mathrm{a}}$, Adrian Nagle ${ }^{\mathrm{a}}$, Rick Ortiz, Tony Kouri ${ }^{a}$, Paul Hauser ${ }^{a}$, Jon Seerveld ${ }^{a}$, Dave Kubalak ${ }^{b}$, Brad Greeley $^{b}$, Claef Hakun ${ }^{b}$, Doug Leviton ${ }^{\mathrm{b}}$, Qian Gong ${ }^{\mathrm{b}}$, Pam Davila ${ }^{\mathrm{b}}$, Ray Ohl ${ }^{\mathrm{b}}$, Jeff Kirk ${ }^{\mathrm{c}}$, Clint Davis ${ }^{\mathrm{c}}$, Jenny Chu ${ }^{\mathrm{c}}$, Erin Wilson ${ }^{\mathrm{d}}$, Bill Chang ${ }^{\mathrm{e}}$, Steve Mann ${ }^{\mathrm{f}}$, Robert Rashford ${ }^{\mathrm{g}}$, Ed Shade $^{\mathrm{h}}$, Corbett Smith ${ }^{\mathrm{i}}$

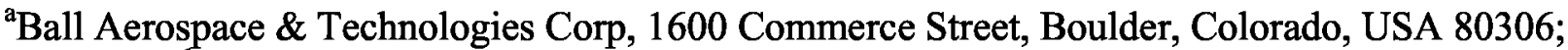
'NASA Goddard Space Flight Center, Greenbelt, Maryland, USA 20771;

'Orbital Sciences Corporation TSD, 7500 Greenway Center Drive, Greenbelt MD 20770;

dStinger Ghaffarian Technologies, 7701 Greenbelt Road, Suite 400, Greenbelt, Maryland 20770;

${ }^{\mathfrak{e}}$ Edge Space Systems, Inc, P.O. Box 310, Glenelg, MD 21737-0310;

f The Hammers Company, 7474 Greenway Center Drive, Suite 710, Greenbelt, MD 20770;

${ }^{\mathrm{g}}$ Genesis Engineering Solutions, Inc, 9811 Greenbelt Road, Suite 102, Lanham, MD 20706;

${ }^{\mathrm{h}}$ Lockheed Martin Corp, 8800 Greenbelt Rd, Greenbelt, MD 20770;

iATK Space Systems, 5050 Powder mill Road, Beltsville, MD 20705

\begin{abstract}
OSIM is a full field, cryogenic, optical simulator of the James Webb Space Telescope (JWST) Optical Telescope Element (OTE). It provides simulated point source/star images for optical performance testing of the JWST Integrated Science Instrument Module (ISIM). OSIM is currently being assembled at the Goddard Space Flight Center (GSFC). In this paper, we describe the capabilities, design, manufacturing and integration status, and uses of the OSIM during the optical test program of ISIM and the Science Instruments. Where applicable, the ISIM tests are also described.
\end{abstract}

Key Words: JWST, Optical Simulator, OSIM, ISIM

\section{INTRODUCTION}

The JWST OTE Optical Simulator (OSIM) is designed to simulate the optical performance of the JWST Telescope. The simulator consists of the primary imaging optical path, which relays optical star sources to the focal surface of the Integrated Science Instrument Module (ISIM). The OSIM generated source images simulate stars over the respective Science Instrument (SI) Field-Of-View (FOV). The wavelength range for the star sources extends from 0.642 microns to 5.6 microns. The OSIM can also simulate up to 14 different pupil configurations.

The design of OSIM allows for initial ambient (293K) testing and also cryo vacuum (100K) optical performance verification for testing ISIM. Mechanisms inside the OSIM Optical Bench Module (OBM) provide alignment compensation capability when going from ambient to cryo vacuum environments.

OSIM consists of the 6 optical modules and subassemblies:

- Optical Bench Module (OBM)

- Alignment Diagnostics Module (ADM)

- Source Delivery Module (SDM)
- Pupil Imaging Module (PIM)

- Beam Image Analyzer (BIA)

- Monitor and Calibration Assembly (MCA)

Each of these modules and subassemblies are discussed in the following sections. Figure 1 shows the layout of the OBM. Integration occurs in the GSFC Space Craft Systems Development and Integration Facility (SSDIF). 


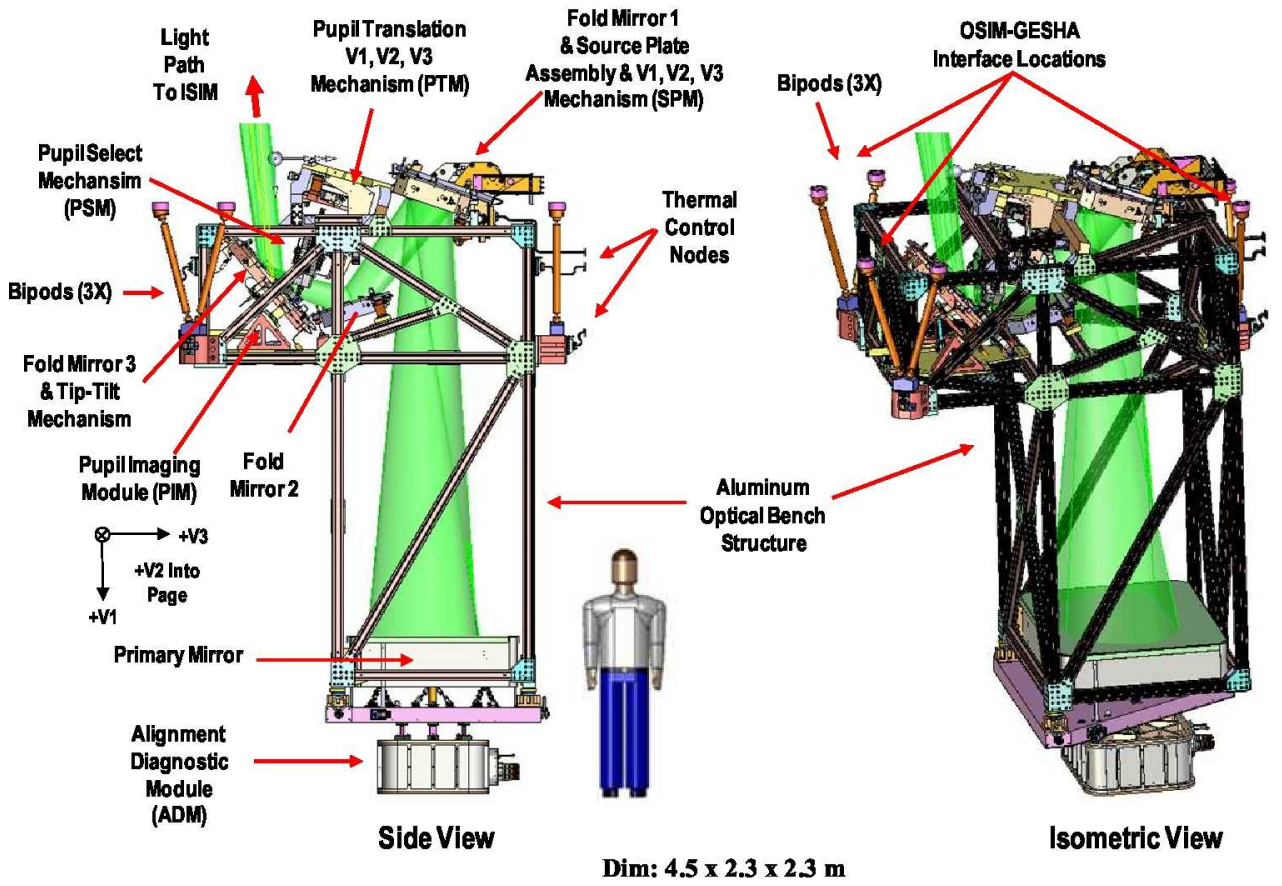

Figure 1. OSIM Optical Bench Module.

OSIM is connected to the ISIM test setup by a set of bipods connected to the Ground Equipment Support Hardware Assembly (GESHA), shown in Figure 2. GESHA not only provides the OSIM mounting interface, but also provides vibration isolation of the entire ISIM/OSIM test configuration in the Space Environmental Simulator (SES) vacuum chamber at the NASA Goddard Space Flight Center. During checkout and calibration, the Beam Image Analyzer (BIA) will be used to measure OSIM image quality and image location relative to a Master Alignment Target Fixture (MATF) located on the Integration Test Platform (ITP) that the ISIM will be mounted to during system level vacuum cryo testing. The BIA will be installed on the ITP in place of the ISIM, during the initial OSIM ambient and cryo checkout and calibration tests.

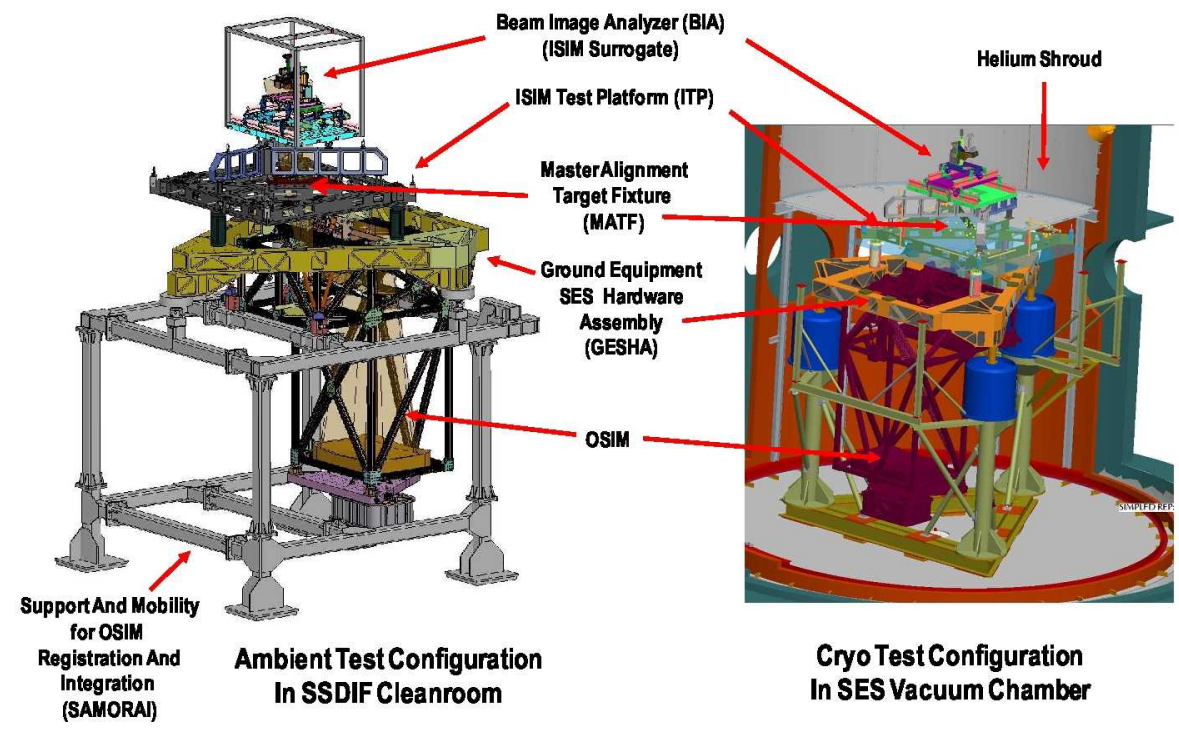

Figure 2. OSIM ambient and cryo test configurations. 


\section{OSIM OPTICAL CONFIGURATION}

OSIM $^{1}$ provides point image inputs for stimulating the JWST science instruments when the optical telescope element (OTE) is not present. It is a finite-conjugate, reflective relay, which images point sources to the SI's object surface (Figure 3). Both source and image surfaces are spherical: The image surface is defined by requirement to match that of the OTE, and the object surface is chosen to be conjugate.

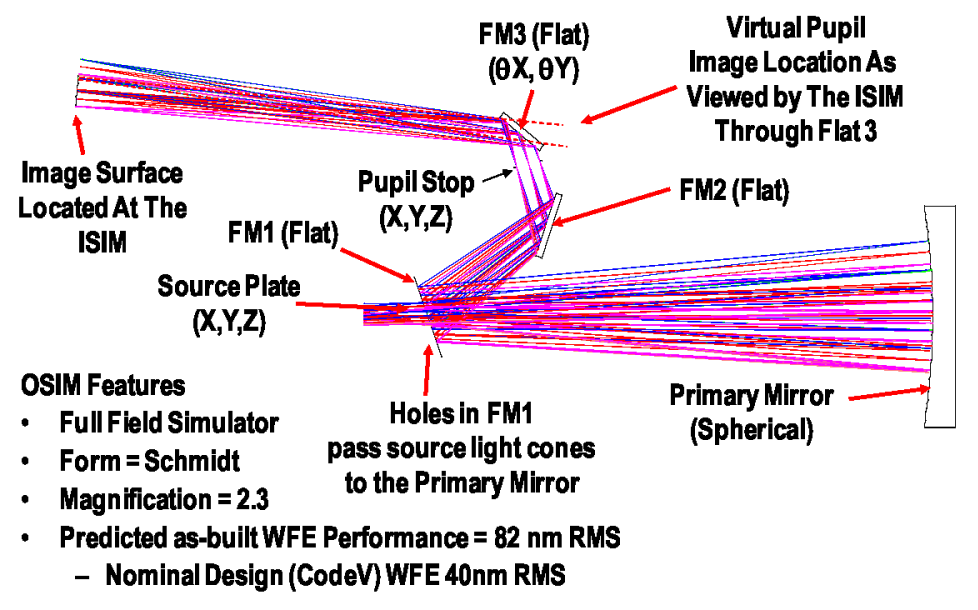

Figure 3. OSIM optical configuration.

OSIM's point sources take the form of either the output of an optical fiber or a back-illuminated pinhole, depending on the target instrument and wavelength. Source ferrules are mounted to a single source plate, and adjusted so that they protrude to the (virtual) object surface. OSIM allows simultaneous stimulation of each SI to facilitate boresight stability testing. This wide field-of-view requirement drives the size of the $1.8 \mathrm{~m}$ (diagonal dimension) primary mirror. A pupil stop between FM2 and FM3 limits the image forming beams to f/20 and provides an exit pupil position which matches that of the OTE.

OSIM's optical design is an on-axis sphere (primary mirror) with the stop near the center of curvature, sometimes referred to as a Schmidt system with the aspheric corrector plate omitted. Its sole powered optic is its spherical primary mirror. The three fold mirrors are used to meet packaging constraints and enable steering of output point images. Onaxis operation is achieved by injecting light from the point sources through small holes in FM1. These holes become obscurations to the rays as they reflect from FM1 downstream of the primary mirror.

The image relayed to each SI can be steered within its field of view allocation using the tip/tilt actuation of FM3. In this mode, the stop must be simultaneously translated to hold the exit pupil position constant. The stop is embodied as a mask located in the dual wheel Pupil Select Mechanism (PSM), just upstream of FM3. The PSM is mounted on a threeaxis Pupil Translation Mechanism (PTM), which provides the required translation ability to maintain exit pupil position during steering. Stop choices include a circular aperture to provide an $f / 20$ beam, a tricontagon (a complex shape representing the outline of the hexagonal segments of the OTE's primary mirror) and numerous other masks to enable testing of ISIM and self-diagnostics of OSIM. Steering of FM3 is also used to direct science instrument sources to diagnostic equipment, including a Point Diffraction Interferometer (PDI) and radiometric detectors, and to direct the line-of-sight of the a Alignment Diagnostics Module (ADM) to metrology targets.

OSIM's $40 \mathrm{~nm}$ RMS of residual design aberrations are dominated by spherical aberration. Since the stop is not quite at the PM center of curvature and is shifted during the course of steering, a small amount of astigmatism develops for offaxis sources. The total RMS wavefront error during operation in cryo-vac conditions is predicted to be less than $82 \mathrm{~nm}$. 


\section{OSIM MODULES AND SUBASSEMBLIES}

\subsection{Optical Bench}

The OSIM optical bench consists of an aluminum truss structure. Each of the members is joined together through gusset plates and each of the joints bolted and pinned (Figure 1). The integrated Optical Bench Module will weigh an estimated $2600 \mathrm{Kg}$. Metrology was conducted on the entire bench structure during the assembly process. A best fit coordinate system was established off of key metrology references locations using a Leica Laser Tracker and removable Spherically Mounted Retroreflectors (SMRs). The three mounting interface locations for the Primary Mirror were fixed and the mounting interfaces for FM1, FM2, FM3 and the PTM were aligned to closely match the CAD Model for the optical bench. Each of the optical elements is interfaced to the optical bench through three point flexure mount assemblies.

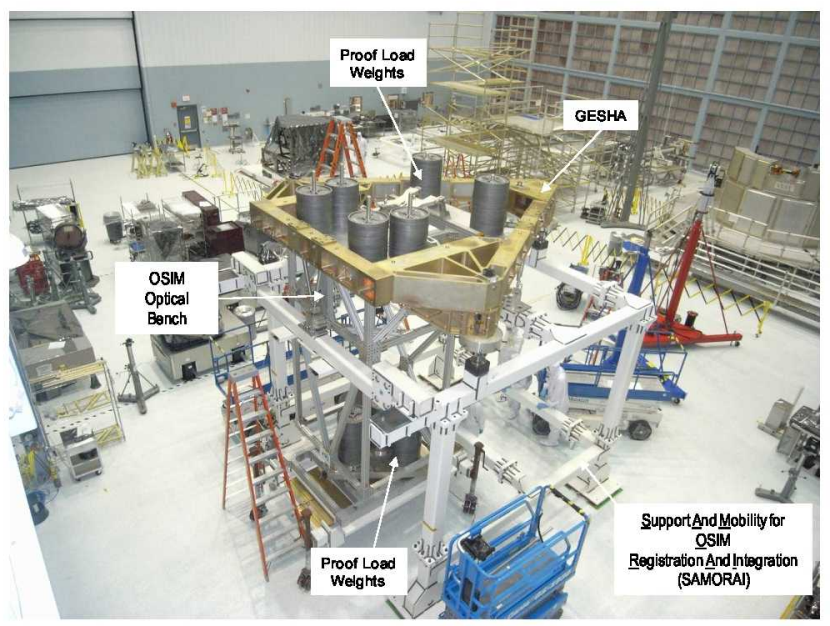

Figure 4. Bench structure proof load test.



Figure 5. Mirrors, mechanisms and ADM in optical bench.

A structural model was developed to assess the bench performance at both $293 \mathrm{~K}$ and also at $100 \mathrm{~K}$ operating temperatures. In addition, this model helps us determine the amount of mechanism motion need for the SPM, PTM and FM3 to correct for cool down alignment shifts.

After the optical bench was shipped from BATC to GSFC, it was installed on the Support And Mobility for OSIM Registration And Integration (SAMORAI) integration structure. A $2 \mathrm{X}$ proof load was successfully conducted to verify structural integrity (Figure 4). The bench then underwent a single thermal cycle from ambient temperature down to 100K. Metrology, using Theodolites, Laser Trackers, and Laser Radar, was conducted on the bench structure during the proof load operation and also pre and post thermal cycle operation. The bench structure behaved as expected under load and also pre and post thermal cycle. Figure 5 shows the mirrors, mechanisms and ADM integrated to the bench.

\subsection{Primary Imaging Optics}

The Primary Imaging Optics consists of a Primary Mirror with a spherical surface and three fold mirrors used to direct the beam to the required image location. The following paragraphs discuss each of the Primary Imaging Optics.

The Primary Mirror is a sixty percent light-weighted $1500 \mathrm{~mm} \mathrm{X} 1120 \mathrm{~mm} \mathrm{X} 200 \mathrm{~mm}$ thick Zerodur substrate with a protected aluminum coated spherical surface having a radius of curvature of $4600 \mathrm{~mm}$. There are three bi-pod flexure mounts attached to three solid Zerodur pads on the back of the primary mirror. The assembly is then fastened to a triangular shaped bulkhead that interfaces to the OSIM Bench. Figure 6 shows the bi-pods attached to the pads on the primary mirror together with the primary mirror mounted to its bulkhead. 

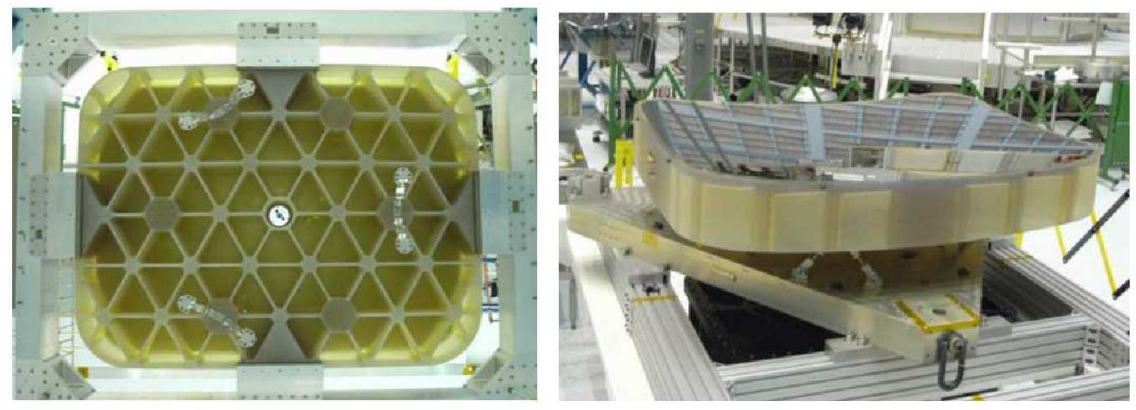

Figure 6. Primary Mirror with attached Bi-Pods and installed on the OSIM bulkhead.

FM1 is a $600 \mathrm{~mm} \times 500 \mathrm{~mm} \times 125 \mathrm{~mm}$ thick Zerodur front surface mirror which operates at 19 degree angle of incidence. To allow the OSIM sources to transmit from their various locations, Fold Mirror 1 has a corresponding set of holes machined into it as shown in Figure 7 below.

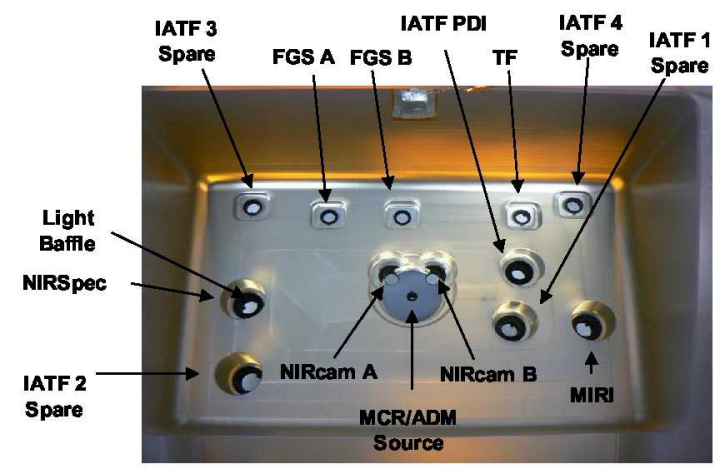

Figure 7. Machined holes in FM1.

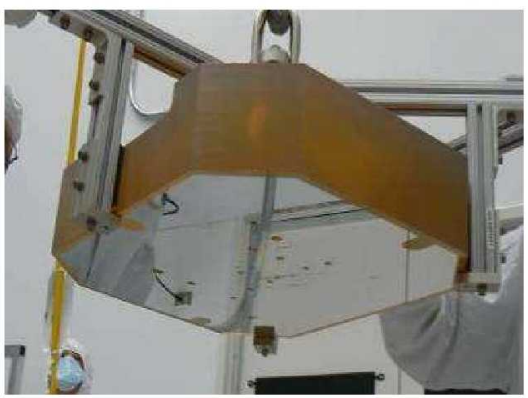

Figure 8. FM1 coated.

Each source hole also has a stray light baffle. The source plate assembly fits into the cutout shown on the back of the mirror. The mirror front surface has a protected aluminum coating (Figure 8). It is currently mounted into its cell and integrated with the Source Plate Mechanism and installed on the OSIM Bench as shown in Figure 5.

FM2 is a $380 \times 475 \mathrm{~mm} \mathrm{X} 90 \mathrm{~mm}$ thick Zerodur front surface mirror that operates at 56 degree angle of incidence in the OSIM system. The mirror has a protected aluminum coating. It is currently mounted in its cell (Figure 9) and installed onto the OSIM Bench as shown in Figure 5.

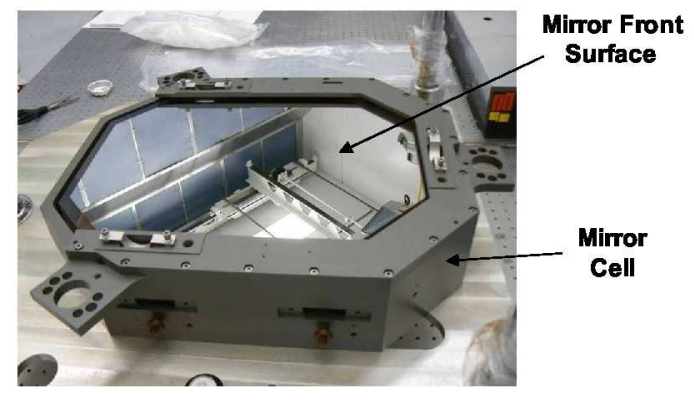

Figure 9. FM2 installed in cell.

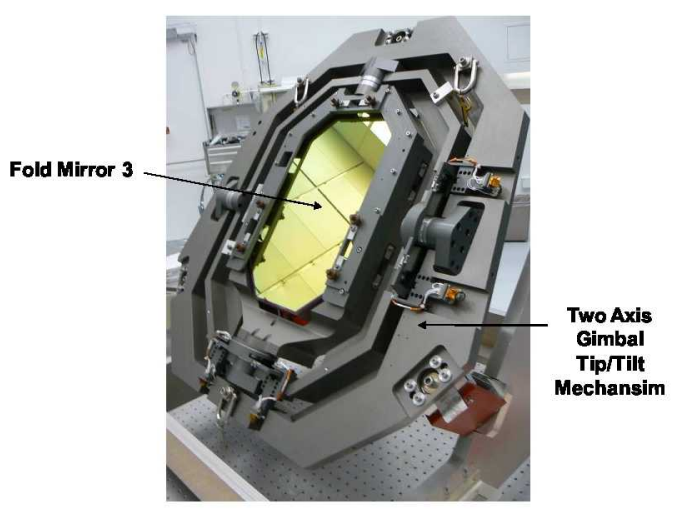

Figure 10. FM3 in Gimbal Assembly. 
FM3 is a unique element. It is designed to be highly reflective at wavelengths beyond $1 \mu \mathrm{m}$, but partially transmissive at $0.633 \mu \mathrm{m}$. As a result it has a fused silica substrate polished on both sides and is anti-reflection coated on the back surface. The front surface has an induced transmission coating making it a beamsplitter at $0.633-0.642 \mu \mathrm{m}$. This supports OSIM's Pupil Imaging Module, which is a detector array that records the return beam when reflective targets in or near the science instrument pupils are deployed. Fold mirror 3 is $300 \mathrm{~mm} \mathrm{X} 440 \mathrm{~mm} \mathrm{X} 80 \mathrm{~mm}$ and operates at a 56 degree angle of incidence.

FM3 is currently mounted in its cell and installed in a two axis gimbal assembly (Figure 10) and installed on the OSIM Bench.

\subsection{Mechanisms}

\subsubsection{Source Plate Mechanism}

The Source Plate Mechanism (SPM) is mounted on the back of FM1 (Figure 11). The SPM positions the source fibers and LED's relative to the master chief ray for the respective SI. It selects one of the 4 fiber or LED sources located in a Super Ferrule assembly, in the source object plane and also positions each source in focus relative to the OSIM/ISIM focal surface. This provides the capability for focus diversity to conduct phase retrieval measurements. The SPM positions the Source Plate Assembly to locate individual fibers or LED sources for the selected SI under test. There are a total of 9 source locations that are used to generate simulated star sources. The SPM is positioned to select one of the fibers or LED's as the source for the specific test to be conducted. The output from each source is injected into the OSIM Primary Imaging Optical Path through the respective hole in FM1. Details of the Source Plate and Source Delivery Module are provided in section 3.5

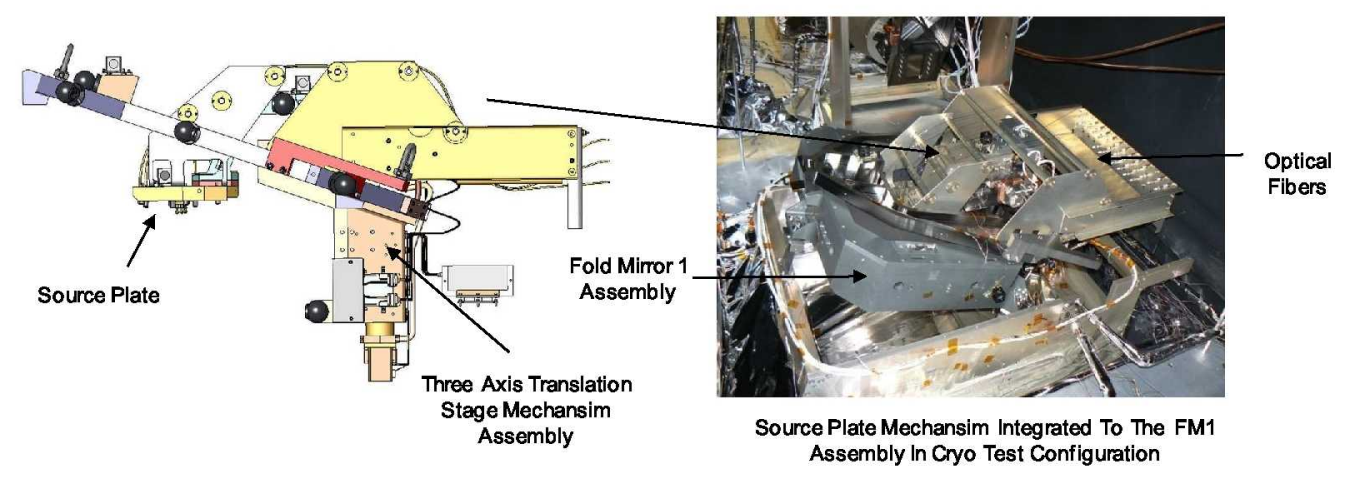

Figure 11. Source Plate Mechanism Assembly.

\subsubsection{Pupil Select Mechanism}

The PSM provides OSIM with the capability to configure the OSIM pupil plane with various optical elements, masks, and alignment reference sources. The two wheels of the PSM each hold 7 optical elements and one open position, and allows for the simultaneous insertion of one element from wheel 1 and one element from wheel 2 into the OSIM pupil plane. The driving performance requirements for the PSM are to operate at $100 \mathrm{~K}$, provide for power off and hold operation, and provide for stable and repeatable optical element placement $(<45$ arcsec $)$. The PSM consists of two nearly identical wheel assemblies. Each assembly consists of a 27 inch diameter wheel which is driven by a direct drive frameless motor. Mechanical detent assemblies provide for the power off and hold and repeatability of the placement of optical elements. An optical encoder provides feedback for the motor controller and optical element position information during operation. Figure 12 shows the major components of the PSM wheels and also the PSM test setup in a dewar prior to the first cold test. The data taken during this test indicates that the PSM is meeting or exceeding its performance requirements 

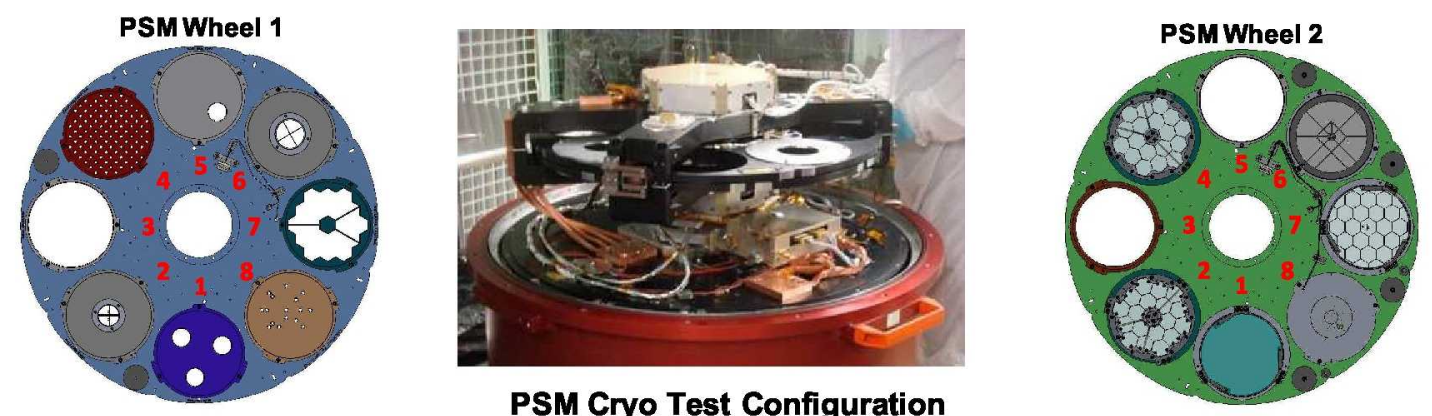

\begin{tabular}{|c|c|c|}
\hline & Descritotion & Punose \\
\hline 1 & JSC \#1 & $\begin{array}{l}\text { Three hole aperture to simulate } 3 \text { phased auto- } \\
\text { collimating flats at the Observatory-level tost }\end{array}$ \\
\hline 2 & PAR \#1 & $\begin{array}{l}\text { Pupil alignment reference for pupil alignment } \\
\text { verification }\end{array}$ \\
\hline 3 & Open & $\begin{array}{l}\text { Open aperture for oversized illumination and } \\
\text { unobscured illumination of wheel } 2\end{array}$ \\
\hline 4 & Distortion & $\begin{array}{l}\text { Mask for testing the NIRCam pupil imag ing lens } \\
\text { optical distortion. }\end{array}$ \\
\hline 5 & JSC \#2 & $\begin{array}{l}\text { One hole aperture to simulate } 1 \text { auto-collimating } \\
\text { flat at the Observatory-level test }\end{array}$ \\
\hline 6 & PAR \#2 & $\begin{array}{l}\text { Pupil alignment reference for pupil alignment } \\
\text { verification. }\end{array}$ \\
\hline 7 & Tricontegon & Mimics the nominal OTE exit pupil obscurations. \\
\hline 8 & Non-degenerate & $\begin{array}{l}\text { Mask for direct measurement of the NIRCam exit } \\
\text { pupil distortion and relative illumination. }\end{array}$ \\
\hline
\end{tabular}

PSM Cryo Test Configuration

\begin{tabular}{|c|c|c|}
\hline & Description & Purpose \\
\hline 1 & Diffuser & Textured sapphire plate for flat-fielding. \\
\hline 2 & $\begin{array}{l}\text { Coarse } \\
\text { Phaseplate }\end{array}$ & $\begin{array}{l}\text { Introduces optical aberrations to simulate } \\
\text { on-orbit coarse phasing observations. }\end{array}$ \\
\hline 3 & $F / 20$ & $\begin{array}{l}\text { Circular aperture that circumscribes the } \\
\text { nominal OTE exit pupil. }\end{array}$ \\
\hline 4 & $\begin{array}{l}\text { Intermediate } \\
\text { Phaseplate }\end{array}$ & $\begin{array}{l}\text { Introduces optical aberrations to simulate } \\
\text { on-orbit intermediate phasing } \\
\text { observations. }\end{array}$ \\
\hline 5 & Open & $\begin{array}{l}\text { Open aperture for oversized illumination } \\
\text { and unobscured illumination of wheel } 1 .\end{array}$ \\
\hline 6 & PSM PAS & OSIM Pupil Alignment Source (PAS) \\
\hline 7 & Fine Phaseplate & $\begin{array}{l}\text { Introduces optical aberrations to simulate } \\
\text { on-orbit fine phasing observations. }\end{array}$ \\
\hline 8 & PSM ADM & $\begin{array}{l}\text { OSIM Alignment Diagnostics Module } \\
\text { (ADM) references }\end{array}$ \\
\hline
\end{tabular}

Figure 12. PSM wheel configurations and cryo test configuration.

\subsubsection{Pupil Translation Mechanism}

The PSM is supported by a Pupil Translation Mechanism (PTM) which is used to adjust the OSIM pupil position perpendicular to and along the OSIM optical axis (Figure 13). This is to maintain the appropriate chief ray angle to the SI under test as FM3 re-points the source images to the selected locations in the respective SI FOV. The PTM consists of three translation stages which position the PSM selected pupil mask in V1, V2 and V3.

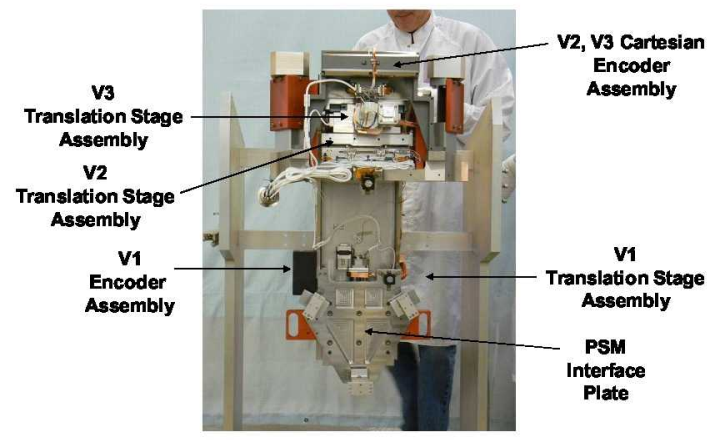

Figure 13. Pupil Translation Mechanism.

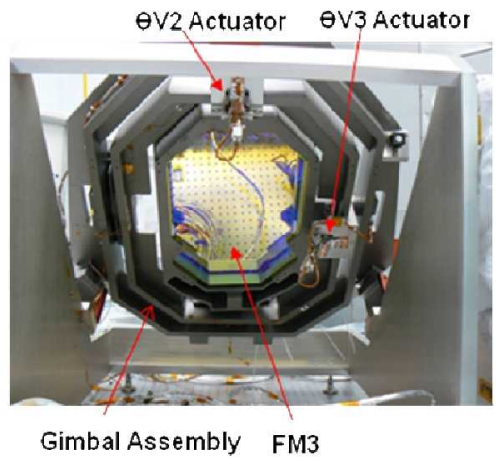

Figure 14. FM3 and Gimbal Mechanism.

\subsubsection{FM3 Gimbal Mechanism}

The FM3 two axis gimbal mechanism (Figure 14) points the OSIM source images over any SI individual FOV. The FM3 provides $\theta \mathrm{V} 2$ and $\theta \mathrm{V} 3$ pointing relative to the OSIM/ISIM focal surface over a range of $+/-5$ degrees in $\theta \mathrm{V} 2$ and $+/-6.75$ degrees in $\theta \mathrm{V} 3$ with a pointing resolution of 1.5 arcsec in each axis. 


\subsubsection{Optical Encoders}

The entire travel of each mechanism axis in OSIM is redundantly encoded with NASA's absolute, pattern recognition encoder. Each encoder head employs a LED and a CCD image sensor which operates down to $75 \mathrm{~K}$. The CCD's are operated by custom, four-channel, cryogenic, differential drive electronics which work down to $100 \mathrm{~K}$. Glass Encoder scales provide a reasonably close thermal expansion match to their titanium mounting parts. Rotary encoders are sensitive to changes in angle to less than 0.3 arcsec rms. Linear encoders are sensitive to motions less than 0.2 microns rms. There are 32 absolute encoders total in OSIM.

\subsection{Alignment Diagnostic Module}

The Alignment Diagnostic Module (ADM) (Figure 15) is located behind the OSIM Primary Mirror, and defines the OSIM chief ray Line-Of-Sight (LOS) through a small hole in the center of the Primary Mirror up to the center of FM1, FM2, the PSM and FM3. The ADM is also used to monitor the LOS pointing of OSIM relative to the test configuration $\mathrm{V}$ coordinate system defined by the MATF. Additional targets are also located on the IATF which mounts on top of the ISIM. The ADM along with the pointing capability of FM3, is used to monitor alignment stability relative to the MATF defined V coordinate system during cool down from room temperature to the OSIM 100K operating temperature and also alignment stability during ISIM testing. The ADM components are enclosed inside a pressure vessel, which maintains room temperature and ambient pressure control.

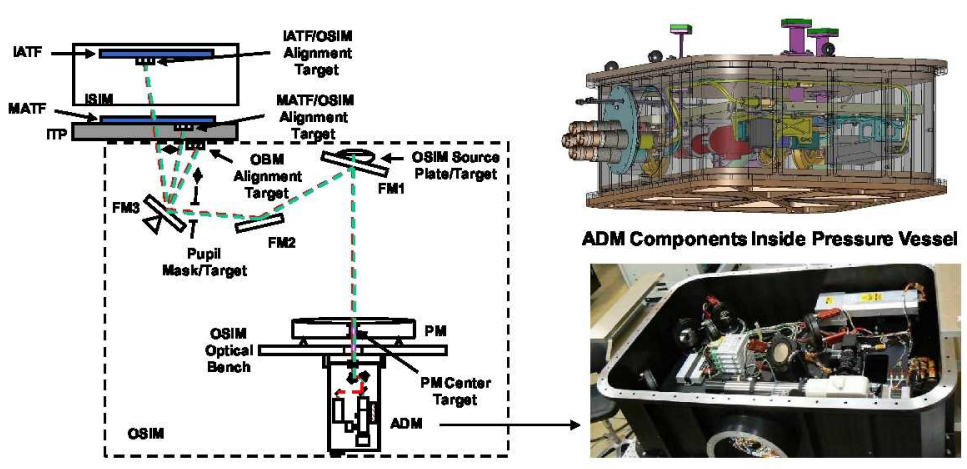

Figure 15 Alignment Diagnostic Module.

\subsection{Source Delivery Module}

The OSIM SDM feeds light to the OSIM source plate. This is accomplished mainly through the use of fiber optics, so that heat sources on the source plate can be limited. The MIRI SI source point is the only one which does not use fibers exclusively, instead relying on LEDs to stimulate MIRI at $5.6 \mathrm{um}$. At each source point, there is a fiber mount containing four fiber ferrules, shown in Figure 16. These are populated as shown in Table I. Using the SPM, The mount can be moved so that one of the four fiber positions is centered behind the hole in FM1 for each source point. Position 1 contains a single mode fiber primarily for use with the OSIM Point Diffraction Interferometer, which uses sources at 642,660 , and $780 \mathrm{~nm}$. Position 2 contains a 200 um core ZBLAN fiber for most of the SI positions, which allows for light from 1-4.5 um. For MIRI, it contains a short light pipe to a filtered LED which provides a $165 \mathrm{~nm}$ FWHM source at $5.6 \mathrm{um}$. For all of the SIs, Position 2 has a pinhole at the end of the fiber which provides for an unresolved source. Position 3 contains a single mode fiber for use with near infrared sources that are fed in from outside the vacuum chamber. The available sources for this fiber include laser diodes at 1.06, 1.32, and $1.55 \mathrm{um}$, as well as a super continuum source for testing the NIRCam DHS in the 1.0-1.7 um band. Position 4 is mainly used as a backup for the ZBLAN fibers, which are very brittle and prone to breakage.

The sources for the ZBLAN fibers reside in a cold $(\sim 100 \mathrm{~K})$ box a few feet away from OSIM. For each pair of primary and redundant ZBLAN fibers, there is a module which uses a small integrating sphere to combine a Helioworks $3.5 \mathrm{~W}$ sapphire windowed tungsten filament lamp and an LED in to the two fibers. Light to the individual fibers is controlled by the use of a fast Uniblitz LS3 shutter, which can be pulsed, or held open. The LED provides a narrower long 
wavelength source at about 1.9 um for the NIRCam SI (for use with the Pupil Imaging Lens), 2.1 um for FGS and TFI, and $3.5 \mathrm{um}$ for the NIRSpec SI. The LED current provides the intensity adjustment. For the tungsten lamps, the intensity can be controlled either by adjusting the lamp current, or by moving an aperture that is place in front of the lens that images the lamp onto the integrating sphere.

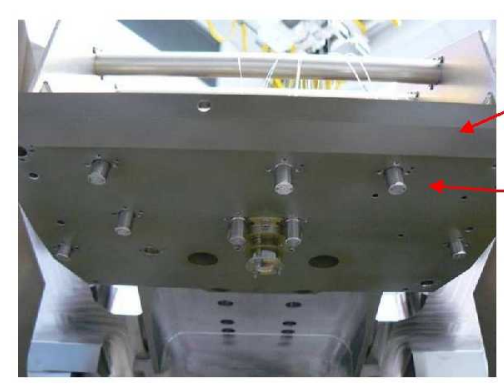

Super Ferrules That Point Through Back of FM1

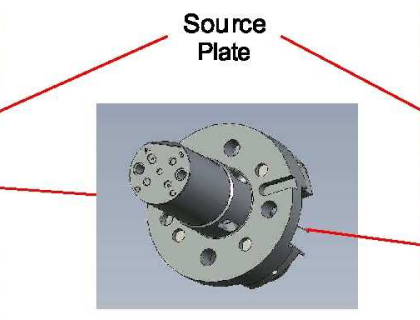

Super Ferrule

4 Optical Fiber/Pin Hole Sounce Locations in Each Super Ferrule

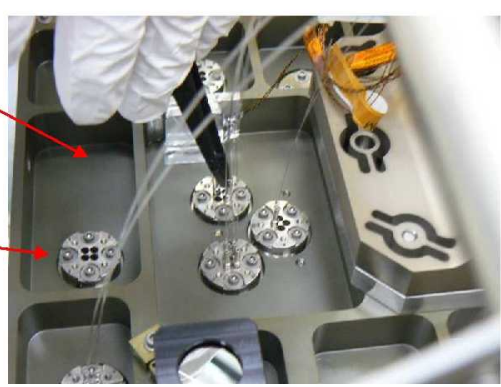

Fiber Installation Into Super Ferrules

Figure 16. Fiber Source Plate Assembly.

Table I. Source Summary

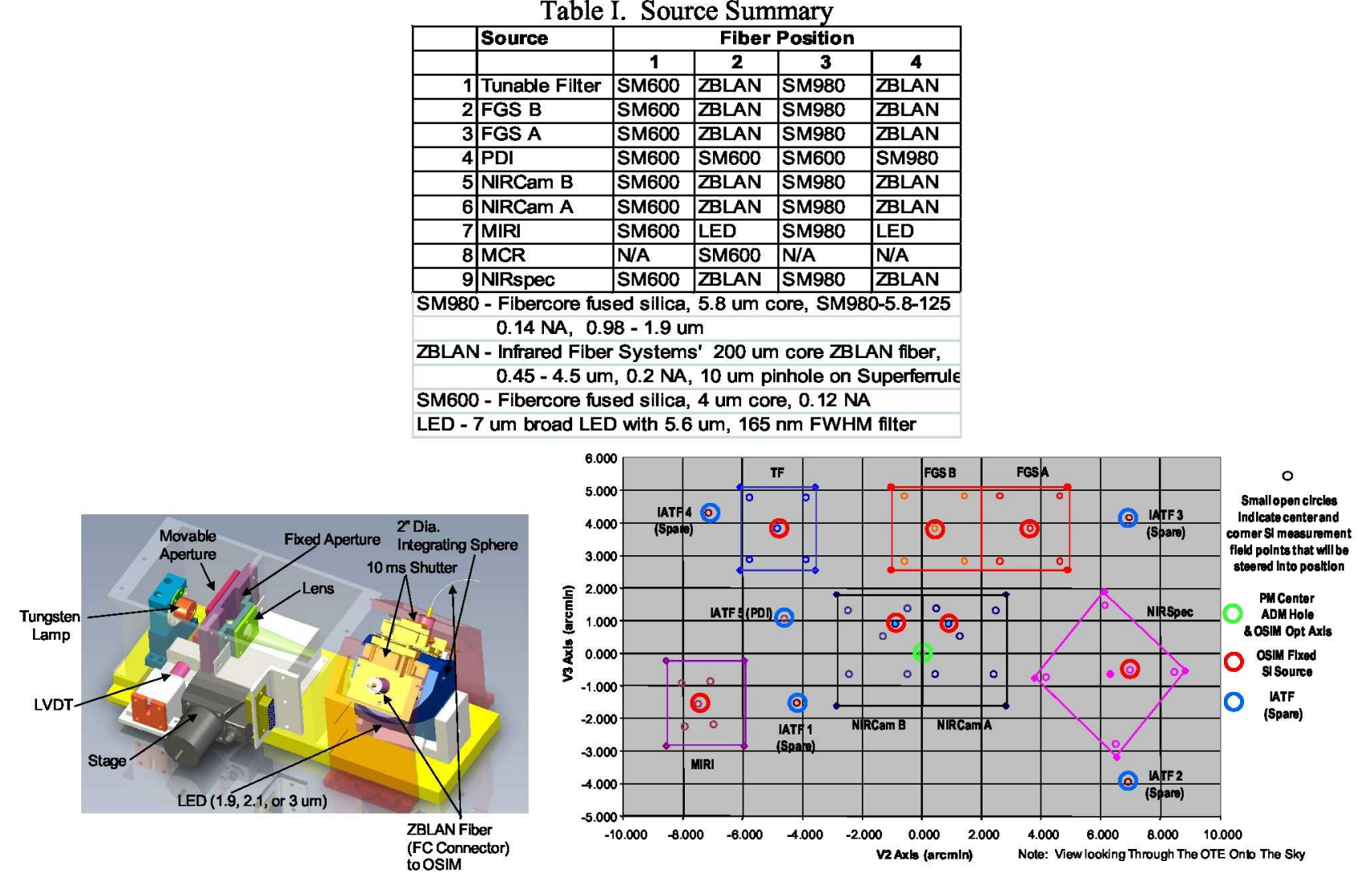

Figure 17. Cold Box source assembly.

Figure 18. OSIM source image field points on the OSIM/ISIM focal surface.

Figure 18 shows the source image pattern that is projected onto the OSIM/ISIM focal surface. There is one fixed source image for each SI located near the center of the SI FOV The fixed field points are steered across the ISIM FOV using a combination of FM3 and Pupil Stop Mechanism motion to set both image location and also chief ray angle. 


\subsection{Pupil Imaging Module}

The PIM (Figure 19) consists of a 4K x 4K CCD detector array located directly behind FM3 which will view visible $0.642 \mu \mathrm{m}$ source light reflecting off of pupil targets located in each of the SIs for comparison to pupil alignment references located in the PSM. This allows measurement of SI to OSIM pupil shear due to alignment offsets in the SI's which may be present after installation into the ISIM structure or due to cool down misalignments.

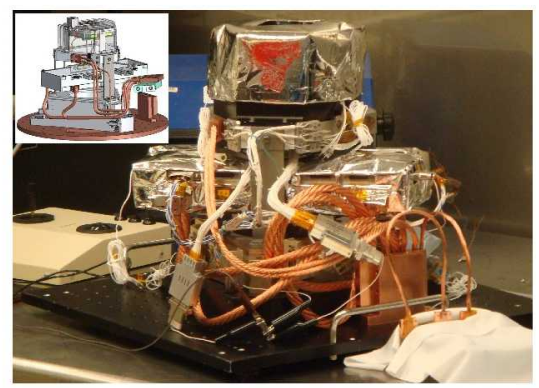

Figure 19. PIM assembly in cryo test.

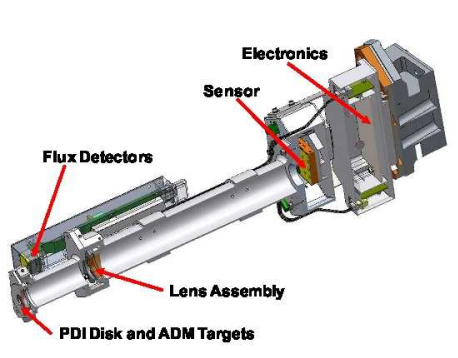

Figure 20. Monitor and Calibration Assembly.

\subsection{Monitor and Calibration Assembly}

The MCA, shown in Figure 20, includes a PDI wavefront sensor, two flux monitors and targets for use with the ADM. It resides inside of the ISIM on the OTE focal surface between the NIRCam and MIRI. It is aligned so that its optical axis is parallel to the master chief ray of the ISIM. Its position and alignment will be measured using the OSIM ADM while it is in situ with the science instruments. During SI testing, the images used to illuminate the instrument will be moved to the PDI position and the OSIM wavefront, chief ray angle pupil illumination will be measured. These data will be used to back out OSIM wavefront from the wavefront calculated for the science instrument at that field point

The flux monitors consist of a $5 \mathrm{~mm}$ Si photodiode and a $7 \mathrm{~mm} \mathrm{InSb}$ photodiode, for measuring source image flux prior to directing the light to the SI's. The Si photodiode, which is sensitive out to about $1.1 \mathrm{um}$, can be used at room temperature to check all of the OSIM fibers before the chamber is pumped down. The InSb can be used from 1-5.2 um.

The PDI assembly includes a PDI disc, an achromatic lens set, and a 2 dimensional detector array. The PDI disc is located at the focal plane of OSIM. The achromat relays the exit pupil onto the detector surface. The beam from each OSIM SI source will be directed to the PDI by tilting FM3 and adjusting the source position. The captured interferogram will be analyzed and the wavefront of the source provided. By moving the source image off the PDI pinhole, a uniformly illuminated pupil is obtained. The chief ray angle will be determined from the center of the pupil and the PDI pinhole position. A more accurate chief ray angle will be obtained using the tilt terms from the analyzed interferogram Zernikes to correct the slight offset between the pinhole and PSF while the interferogram is taken.

\subsection{Beam Image Analyzer}

The BIA consists of an optical sensor head mounted on a three-axis translation stage assembly mounted above the OSIM (Figures 2 and 21). The translation stage assembly allows the sensor head to be moved accurately over the entire OSIM field of view (FOV) to permit accurate measurement of the OSIM image and pupil parameters at any desired point in the FOV. The translation stages are encoded with high accuracy absolute encoders developed in the Optics Branch at the Goddard Space Flight Center to measure the position of the sensor head in the OSIM Field of view. The sensor head contains a phase retrieval camera (PhaRet) and a PDI as well as two flux monitors and targets for measurement of its position using the OSIM internal ADM. The PhaRet will collect focus-diverse images produced by OSIM. The PDI will collect interferograms of the OSIM wavefront to determine OSIM wavefront, chief ray angle and other pupil parameters. The PDI is also mounted on an encoded tilt stage to allow accurate measurement of the chief ray angle for each image point. Both of these sensors have ambient and cryogenic forms. These forms differ in the detectors used to collect data. The ambient form uses a scientific grade CCD as the detector. The cryogenic form uses NIRCam H2RG detectors and electronics. 

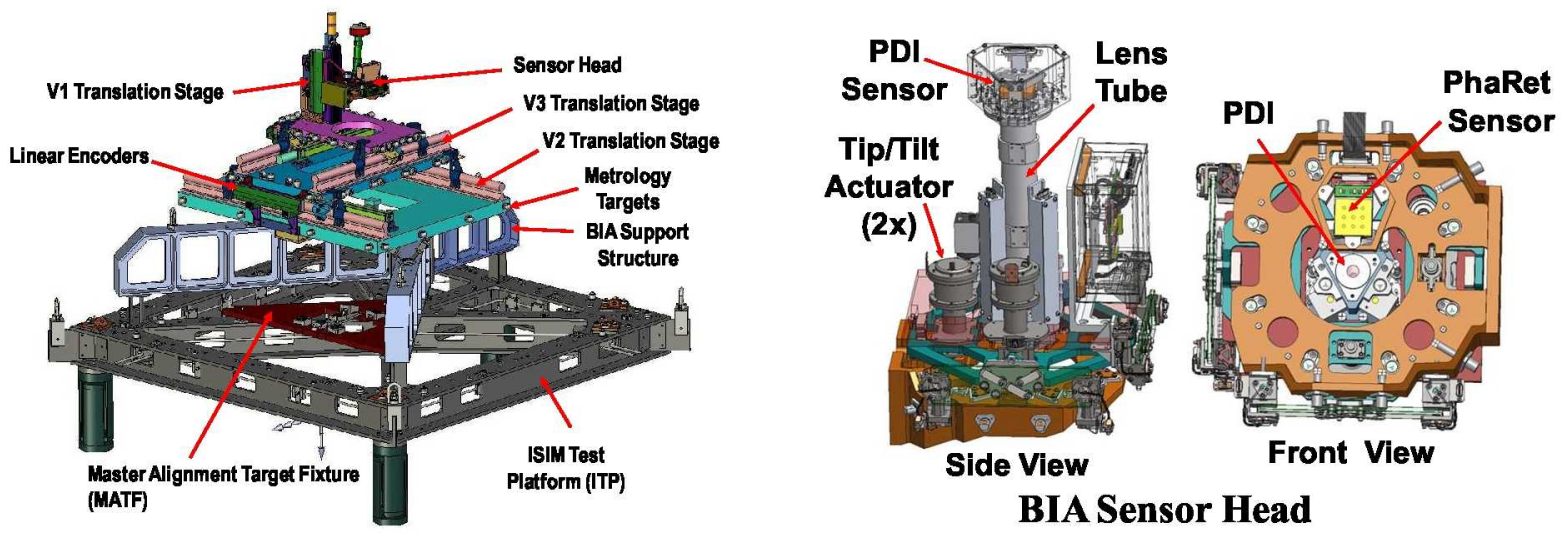

Figure 21. BIA and Sensor Head with PhaRet and PDI.

\section{OSIM TEST PROGRAM OVERVIEW}

The OSIM will be used as the Master Tool to demonstrate performance and specification compliance of the ISIM Element for the JSWT program. It represents the only point in the JWST test program during which the SIs will produce images similar to those expected on orbit. Using OSIM, science instrument wavefront quality, boresight and coboresight stability, pupil co-alignment, focus, wavefront sensing performance, and multi instrument multi field (MIMF) capability will be verified in an operational environment.

The main objective of OSIM is to present the SIs with images that represent those expected on-orbit from the JWST Telescope. These images must have known aberration content. Their location on a focal surface with respect to a welldefined global datum must be known. Their boresight and exit pupil location with respect to this datum must also be known. The test program for OSIM must demonstrate that OSIM can satisfy these requirements when the ISIM is in its operating environment. The validation program must therefore include at a minimum: image/wavefront quality and knowledge determination, global image location accuracy and knowledge determination, focal surface location and orientation and chief ray/pupil position determination.

The verification campaign for OSIM will be conducted in 4 phases after the completion of OSIM assembly and alignment. The first two phases are ambient tests of the OSIM optical system and the metrology system. The first of these will be in the SSDIF. The second will be in the SES chamber. The second two phases will be performed with OSIM at its cryogenic operating temperature of 100K. The first one will be OSIM alone at operational temperature in the SES. The final OSIM verification will be an in-situ verification check with the ISIM in the SES chamber.

For the most part, the testing will use the BIA as the primary tool. The optical metrology portion of the verification program will be done by photogrammetry (PG). The final check of OSIM's performance in-situ with ISIM and the SI's will use the MCA.

One facet of the OSIM calibration is redundancy. Where possible, each OSIM requirement is verified by more than one method. This will allow self cross checks to be in place. For example, aberration content at each field point will be determined both by Phase Retrieval as well as interferometry. The position of the sensor head will be determined by the $\mathrm{ADM}$ as well as by the BIA encoders. Image aberrations, encircled energy and position will be measured by the PhaRet. Aberrations will be determined by Phase Retrieval Analysis of focus diverse images. Two software packages are planned for this determination. The ASPRIN software package that was developed for use by the Hubble Independent Verification team is one. The other is the Hybrid Diversity Algorithm (HDA) with Variable Sample Mapping (VSM) developed by the Wavefront Sensing and Control Group in the Optics Branch at Goddard Space Flight center ${ }^{2,3}$. Image positions will be determined my metrology of the sensor head location with respect to the ISIM coordinate system as defined by the MATF and the ISIM Alignment Target Fixture (IATF) using the BIA's internal high-accuracy encoders as well as the OSIM's internal ADM. Photogrammetric targets on the BIA sensor head will allow external calibration of its position with respect to the ISIM coordinate system that can be used to verify the ADM calibration. For the 
metrology verification, optical targets on the sensor head are measured by the PG system and by the ADM. The measurement points of the PhaRet and the PDI are known with respect to these references. The MATF targets are also measured by the ADM and PG. Knowledge of these target positions in the ISIM coordinate system allows a transformation of the PhaRet and PDI measurement points into the ISIM coordinate system. Agreement between the $\mathrm{ADM}$ and $\mathrm{PG}$ allows a verification of the ADM performance.

In order to meet the tight calibration requirements levied on OSIM for wavefront knowledge, the PhaRet and the PDI have to be separately calibrated. The PhaRet and the PDI will be calibrated at temperature at GSFC in an ad hoc facility developed to perform this work. The facility consists of an off-axis ellipse and aperture that produces a JWST-like, $\mathrm{f} / 20$, beam. The aberration content of the beam will be variable to allow the measurement of the PhaRet and PDI response to differing aberrations. The facility will be calibrated by various tools all of which have been proved during the Hubble era. They are the Aberrated Beam Analyzer (ABA), the Goddard Phase Retrieval Camera (GOPHER), and an ambient Point Diffraction Interferometer (PDI). Additionally, the Ellipse facility will be characterized with a PhaseCam interferometer. The calibration facility is set up so that, during the PhaRet or PDI calibration, the optical beam can be sequentially measured with the ABA, GOPHER and PDI as well as the detector under test. The PhaRet and PDI will be located in a Dewar for these measurements.

\section{SUMMARY}

The OSIM is a critical tool in the verification and qualification of the Science Instruments and ISIM for the JWST mission. It is the Master Tool that will bless the ISIM and SIs on their way to being a working, world-class observatory. The testing of the Science Instruments with OSIM at NASA's Goddard Space Flight Center will be the only place in the entire test program for this observatory where the instruments will be able to work together in an environment that simulates that expected on-orbit. The OSIM will provide high quality images that will simulate those to be produced by the JWST Optical Telescope. The images will have sufficiently well known optical quality that will allow quantitative determination of the optical performance and alignment of each of the SIs in the ISIM as well as the ISIM's optical performance. In addition to providing a high quality optical calibration, it will facilitate early detection of problems and issues with the co-aligned instruments such as co-bore sight stability, repeatability, thermal stability, pupil shear, and allow a rough determination of sensitivity, alignment stability and trending through its environmental test program. It will ensure that when the ISIM moves on to its final testing at JSC, it will have been qualified to specification and work as planned and allow the JSWT mission to meet its lofty scientific goals.

\section{ACKNOWLEDGEMENTS}

We would like to acknowledge the contributions of Schott for manufacturing the Primary Mirror substrate and ITT for polishing and coating of the mirror. The Fold Mirror substrates were manufactured and polished by Zygo with aluminum coatings for FM1 and FM2 provided by Quantum. The induced transmission coating for FM3 was produced by BARR Associates. The cryo translation mechanisms were manufactured by ATK Mission Research.

\section{REFERENCES}

\footnotetext{
${ }^{1}$ P. S. Davila, et al., "The Optical Telescope Element Simulator for the James Webb Space Telescope," Proc. SPIE 7010 (2008).

${ }^{2}$ B. H. Dean, D. L. Aronstein, J. S. Smith, R. Shiri, and D. S. Acton, "Phase retrieval algorithm for JWST Flight and Testbed Telescope," Proc. SPIE 6265, 626511 (2006)

${ }^{3}$ J. S. Smith, D. L. Aronstein, B. H. Dean, and D. S. Acton, "Phase retrieval on broadband and under-sampled images for the JWST testbed telescope," Proc. SPIE 7436, 74360D (2009)
} 